\title{
Theoretical Calculation Method for the Hugoniot Elastic Limit of Hard Rock Based on Ideal Elastoplastic Model
}

\author{
Yihao Cheng $\mathbb{D}^{1}{ }^{1}$ Chunming Song, ${ }^{1}$ Yizhong Tan, ${ }^{1}$ Songlin Yue, ${ }^{1}$ Gan Li, ${ }^{1,2}$ Yanyu Qiu, ${ }^{1,2}$ \\ Tianhan Xu $\mathbb{D},{ }^{1}$ Yong Liu, ${ }^{1}$ Fei Gao $\mathbb{D}^{2},{ }^{2}$ and Qian $Y u^{3}$ \\ ${ }^{1}$ Army Engineering University of PLA, Nanjing 210007, China \\ ${ }^{2}$ Nanjing University of Science and Technology, Nanjing 210094, China \\ ${ }^{3} 95338$ Troops of PLA, Guangzhou 510405, China
}

Correspondence should be addressed to Yihao Cheng; 05105432@163.com

Received 29 December 2020; Revised 31 August 2021; Accepted 4 October 2021; Published 12 November 2021

Academic Editor: Leilei Niu

Copyright ( 2021 Yihao Cheng et al. This is an open access article distributed under the Creative Commons Attribution License, which permits unrestricted use, distribution, and reproduction in any medium, provided the original work is properly cited.

To study the theoretical calculation method of the HEL for hard rock, the major factors determining the strength of rock under dynamic loadings are firstly discussed. Secondly, the calculation method of the HEL of hard rock is suggested based on the Lundborg strength criterion, and the parameters influencing the HEL are analyzed and discussed. Thirdly, the HEL obtained by theoretical calculation, numerical modeling, and experiments are compared. Fourthly, the abnormal decreasing or increasing of the HEL in plate impact experiments of hard rock is explained. This research shows that the HEL increases with Poisson's ratio, the shear strength at zero pressure, the coefficient of internal friction and the plastic limit, and Poisson's ratio and the plastic limit could be the most important factors. The simplified model of this work can give a rational and practical prediction of the HEL of hard rock in theory, and the complicated interaction between the "damage front" and the diffuse elastic precursor is assumed to explain two special effects of the HEL in plate impact experiments of hard rock, where Poisson's ratio related to damage levels seems to be the dominant factor.

\section{Introduction}

The Hugoniot elastic limit (or HEL, for short) is a fundamental dynamic material property of solid materials, and it is important to study stress wave propagation and evolution of internal damage of materials under explosion and high velocity impact effects [1-5]. The HEL is believed to represent the maximum stress that a rock or other solid materials can withstand under conditions of rapid onedimensional compression without internal rearrangement taking place in the material at the shock front.

Values of HEL have been investigated experimentally using a variety of techniques including explosive detonation loading and flyer-plate impact studies. In these studies, velocity interferometer for any reflector (or VISAR, for short), displacement interferometer for any reflector (or DISAR, for short), and embedded manganin pressure gauges were used to measure the free surface particle velocity or the normal stress parallel to the propagation direction of the shock wave, and the amplitude of the first shock wave front, which has the greater propagation velocity, is the HEL [5-7].

It should be noted that the relation between the HEL and the yield strength of elastoplastic metallic materials has a simple form considering Tresca yield criterion, where only Poisson's ratio and yield strength are required [3]. However, such a simple formula connecting the HEL and other material properties of rock has not been suggested explicitly, and this, to a great extent, should be attributed to the complicated behaviors of rock materials under dynamic loadings. For better understanding of rock dynamics, we suppose that the mechanism analysis of measured HEL, which could even be over simplified, is very helpful, and it is necessary to develop a quantitative relation between the HEL and other rock properties.

In this study, the major factors determining the strength of rock under dynamic loadings are firstly discussed, then 
the calculation method of the HEL of hard rock is suggested based on the Lundborg strength criterion, and the parameters influencing the HEL are analyzed and discussed. To verify the rationality of the HEL obtained by theoretical calculation, numerical modeling and experiments are compared. The abnormal decreasing or increasing of the HEL in plate impact experiments of hard rock are also explained with this method and assumption of the interaction between the "damage front" and the elastic precursor.

\section{Theoretical Estimation of HEL of Hard Rock}

2.1. Factors Influencing the Rock Dynamics. The stress state of the specimen behind the shock front is in the condition of rapid one-dimensional compression as shown in Figure 1, and this cube is compressed by pressure from the top surface with other faces constrained. We can distinguish at least two cases as follows. In the first case, $\sigma_{\mathrm{x}}$ is low enough, and the elastic relation between $\sigma_{\mathrm{x}}, \sigma_{\mathrm{y}}\left(\right.$ or $\sigma_{\mathrm{z}}$ ), and $v$ holds as

$$
\sigma_{y}=\sigma_{z}=\frac{v}{1-\nu} \sigma_{x} \text {. }
$$

In the second case, $\sigma_{\mathrm{x}}$ is high enough, and the difference between $\sigma_{\mathrm{x}}$ and $\sigma_{\mathrm{y}}$, if Tresca criterion holds, stays as a constant, i.e.,

$$
\sigma_{y}-\sigma_{x}=Y=2 \tau_{s}
$$

As a critical value, $\sigma_{\mathrm{x}}$ equals HEL when the above two cases coincides, and it can be explained as an intersection of the uniaxial strain loading path with the strength surface. By combining equations (1a) and (1b), the HEL is calculated as

$$
\mathrm{HEL}=\frac{1-v}{1-2 v} Y .
$$

Experiments on shock-loaded metals have shown that relation (2) works well with $\nu=1 / 3$ [3].

On the other hand, relation (2) cannot be well applied for rock and many other geological materials as $Y$ could change obviously with varying pressure, strain rate, temperature, specimen size, and other factors. To describe how the HEL depends on rock properties, the strength of rock material as a function of the above factors is required.

Although many semiempirical equations, e.g., the $\mathrm{JH}-2$ model for predicting dynamic strength, which include multifactors, is popular in numerical modeling, and they could be unreliable when the environmental parameters are out of the scope where the semiempirical constants hold. We believe that simple but physically based models are also required for substantial understanding of the HEL. Thus, the influencing factors of rock dynamics should be discussed firstly.

2.1.1. Pressure. Multiaxial loading tests show that the yield strength for intact rock rises as pressure (or confining stress) increases, and the strength first increases very rapidly when pressure (or confining stress) is applied, but then slowly saturates at higher pressure (or confining stress). This law is considered in commonly used Hoek-Brown criterion [8]

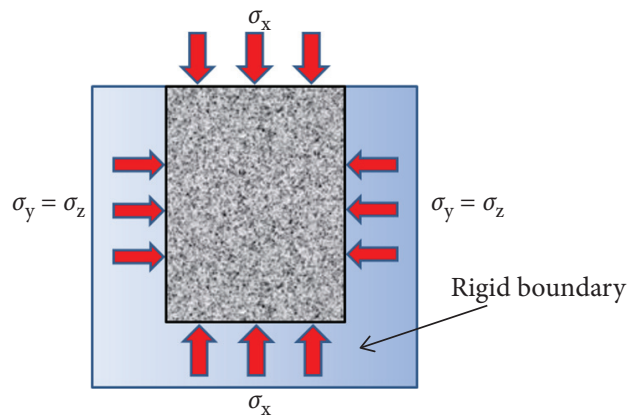

FIGURE 1: Compression with uniaxial strain condition.

and many other semiempirical constitutional models for rock and concrete materials [9-11]. For example, the Hoek-Brown criterion shows that the intact rock strength obeys the following equation:

$$
Y=\sigma_{x}-\sigma_{y}=\sigma_{U C}\left(m \frac{\sigma_{y}}{\sigma_{U C}}+1\right)^{1 / 2}
$$

As shown in equation (3), although the Hoek-Brown criterion can describe the saturation of strength under high confining stress, $Y$ is not actually limited and can increase towards infinity. Such an unlimited trend of strength growing could be unrealistic when the strength model is used in studies considering extremely high pressure. Taking Kuru Gray granite for example with $\sigma_{\mathrm{UC}}=230 \mathrm{MPa}$, $m=27.39$, and $\sigma_{\mathrm{y}}=20 \mathrm{GPa}$ as suggested in reference [12], $Y$ can be calculated to be $11.2 \mathrm{GPa}$, and this is too high compared with its Young's modulus (60 GPa).

The Lundborg criterion [13] is more widely used for rock modeling in researches as a formation of large impact craters [9] and mechanical effect of underground nuclear explosions [14], and it is defined as

$$
\begin{aligned}
\frac{\sigma_{x}-\sigma_{y}}{2} & =\tau_{s}=\tau_{0}+\frac{\mu_{s} P}{1+\left(\mu_{s} P / \tau_{d}-\tau_{0}\right)}, \\
P & =\frac{\sigma_{x}+2 \sigma_{y}}{3} .
\end{aligned}
$$

Compared with the Hoek-Brown criterion, the Lundborg criterion assumed that, at some large confining pressure, the shear strength smoothly reaches its upper limit $\tau_{\mathrm{d}}$.

2.1.2. Strain Rate. The effect of the strain rate on the mechanical behavior of various rocks has been widely studied in uniaxial compressive and triaxial compressive conditions $[2,11,12]$, and it is found that both dynamic uniaxial compressive strength and dynamic triaxial compressive strength increase with increasing strain rate. Studies of Grady [2] show that the failure stress of brittle solids is very sensitive to strain rate within a certain region. However, tests failed to identify significant strain-rate-dependent strength in the high strain rate region with or without external confinement. Tests of Hokka et al. [12] and Li et al. [15] for granite show that the rate of strength enhancement with strain rate is lower at higher confining pressures, and this is 
consistent with the studies of Ai et al. [10] for granite, where pressure varies from 0 to $4 \mathrm{GPa}$ and strain rate varies from $10^{5}$ to $10^{-4} \mathrm{~s}^{-1}$. On the other hand, variation of dynamic differential stress with confining pressure at different strain rates by Sato et al. [16] shows that the dynamic strength curve relative to confining pressure is almost parallel to the static one for each rock type.

2.1.3. Temperature. The shear strength of rock drops obviously near the melting temperature. However, if the propagation of shock wave is fast enough, the heat caused by the impact process and entropy increase is restrained in the local region behind the elastic precursor, and it can hardly affect the macroscopic strength of rock there.

2.1.4. Specimen Size. There is evidence that natural rock materials are much weaker on scales of tens to hundreds of meters with respect to laboratory strength measurements of centimeter-scale rock samples [9]. Numerical simulations of Collins et al. [9] and Yan et al. [17] show that these size effects seem to be attributed to the heterogeneity and localization of deformation, and even at larger scales, the lower limit of rock strength must still be controlled by Coulomb's frictional law. It can be inferred that the strength criterion found under laboratory strength measurements can also be used in larger scales if the strength is reduced accordingly.

As the limitation of the authors' knowledge, not all factors influencing rock strength are considered above. However, for the study of HEL with high confining stress in shock wave propagation, pressure could be the dominant factor of rock strength, and effects of strain rate, specimen size, temperature, and other factors can be considered by increasing or reducing cohesive stress, plastic limit, and friction angle. As the Lundborg criterion is more proper than the Hoek-Brown criterion under high pressure, we will use the Lundborg criterion, i.e., equation (4) to calculate HEL.

2.2. The HEL of Rock with Lundborg Criterion. To calculate HEL with the Lundborg criterion, equation (4) is used to replace equation (1b), while equation (1a) still holds for the elastic state. By combining equations (4) and (1a), it is derived that

$$
A(\mathrm{HEL})^{2}+B \cdot \mathrm{HEL}+C=0
$$

where

$$
\begin{aligned}
& A=\frac{\mu_{s}(1+\nu)}{3\left(\tau_{d}-\tau_{0}\right)} \frac{(1-2 \nu)}{(1-\nu)^{2}}, \\
& B=\frac{1-2 \nu}{1-\nu}-\frac{\mu_{s}(1+\nu) 2 \tau_{0}}{3(1-\nu)\left(\tau_{d}-\tau_{0}\right)}-\frac{2 \mu_{s}(1+\nu)}{3(1-\nu)}, \\
& C=-2 \tau_{0} .
\end{aligned}
$$

It is solved that

$$
\mathrm{HEL}=\frac{-B+\sqrt{B^{2}-4 A C}}{2 A} .
$$

According to equations (5a) and (6), HEL of intact rock material is a function of $\tau_{0}, \mu_{\mathrm{s}}, \tau_{\mathrm{d}}$, and $\nu$, which all should be obtained under dynamic compressive conditions.

To obtain specific results of HEL, $\tau_{0}, \mu_{\mathrm{s}}$, and $\tau_{\mathrm{d}}$ are determined with data of three types of granite firstly suggested by Lundborg [13] as shown in Table 1, where $v$ is assumed to vary in the range of 0.15 to 0.45 . Values of HEL versus Poisson's ratio are shown in Figure 2. It seems that the HEL of different types of rock are quite close to each other with Poisson's ratio the same, and this implies that HEL does not change obviously under slight change of $\tau_{0}$, $\mu_{\mathrm{s}}$, and $\tau_{\mathrm{d}}$. However, the HEL is quite sensitive to Poisson's ratio, and it increases rapidly with $v$ rising. To make predicted HEL close to measured results of Petersen [18] (4.5 GPa), Yuan et al. [5] (3.2-3.5 GPa), and Li et al. [7] $(2.36-2.63 \mathrm{GPa}), v$ should be restrained within the range $0.25-0.38$. This range is generally consistent with results by Li et al. [15] $(\nu=0.25-0.35)$ and Ai et al. [10] $(\nu=0.29)$. A further discussion of Poisson's ratio will be conducted in Section 4.

The parameters of Table 1 are fitted with quasistatic compression tests of rock. Therefore, strain rate effects are not considered in Figure 2. If we attribute the increasing of yield strength to the enhancement of $\tau_{0}, \mu_{\mathrm{s}}$, and $\tau_{\mathrm{d}}$ under dynamic loadings, the strain rate effects on the HEL can be estimated with the sensitivity analysis, and this is made in $s$ (Figures 3(a)-3(c)). It should be noted that, as an inference of Section 2.1, $\tau_{0}$ is much more sensitive to strain rate than $\mu_{\mathrm{s}}$, and $\tau_{\mathrm{d}}$. Therefore, the maximum of $\tau_{0}$ in Figure 3(a) is set to be 5 times of the minimum of $\tau_{0}$, while the maximum of $\mu_{\mathrm{s}}$ and $\tau_{0}$ is set to be 1.22 and 1.5 times of minimum of $\mu_{\mathrm{s}}$ and $\tau_{0}$ in Figures 3(b) and 3(c), respectively.

It can be seen that the HEL almost linearly increases with rising $\tau_{0}, \mu_{\mathrm{s}}$, and $\tau_{\mathrm{d}}$ within the set ranges, and it is more sensitive to $\tau_{\mathrm{d}}$ than $\tau_{0}$ and $\mu_{\mathrm{s}}$. Specifically, the HEL increases $47 \%$ when $\tau_{\mathrm{d}}$ rising $50 \%$, and it only increases $5 \%$ and $6.9 \%$ when $\mu_{\mathrm{s}}$ rising $22 \%$ and $\tau_{0}$ rising $400 \%$, respectively. This implies that, besides Poisson's ratio, $\tau_{\mathrm{d}}$ is also an important factor in determining the HEL, while the influence of $\tau_{0}$ is ignorable. It should be emphasized that $\tau_{\mathrm{d}}$ is the theoretical shear strength when pressure is infinitely great, and this implies that the HEL of hard rock mainly depends on its upper limit ability to resist shear but not those under specific conditions. It can also be inferred that the high strain rate and other factors cannot change the HEL obviously unless they do influence $\tau_{\mathrm{d}}$ or Poisson's ratio greatly. One might doubt the facticity of $\tau_{\mathrm{d}}$ in Table 1 as they are as high as some high-strength alloys. Actually, according to the experimental studies of Shock et al. [19], Hokka et al. [12], and Millett et al. [6], the measured shear strengths of granodiorite, granite, and gabbro are as high as $800 \mathrm{MPa}$ (strain rate of $10^{-5} \mathrm{~s}^{-1}$ ), $700 \mathrm{MPa}$ (strain rate of $600 \mathrm{~s}^{-1}$ ), and $1.95 \mathrm{GPa}$ (shocked state) respectively. Hence, it seems that the ultimate ability of hard rock to resist shear is so high that those values of $\tau_{\mathrm{d}}$ in Table 1 can even be underestimated. 
TABle 1: Parameters of Lundborg criterion [13].

\begin{tabular}{lccccc}
\hline No. & Material & Locality & $\mu_{\mathrm{s}}$ & $\tau_{0}(\mathrm{MPa})$ & 60 \\
1 & Granite I & Bohuslän & 2.0 & $\tau_{\mathrm{d}}(\mathrm{MPa})$ \\
2 & Granite II & Bredseleforsen & 2.0 & 40 & 970 \\
3 & Granite III & Rixö & 1.8 & 30 & 1020 \\
\hline
\end{tabular}

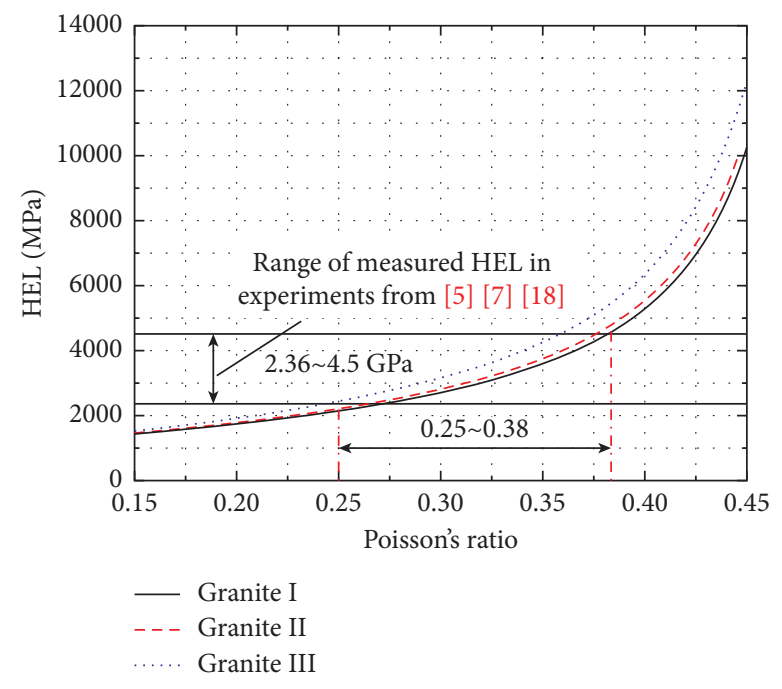

Figure 2: Comparison of calculated and measured HEL.

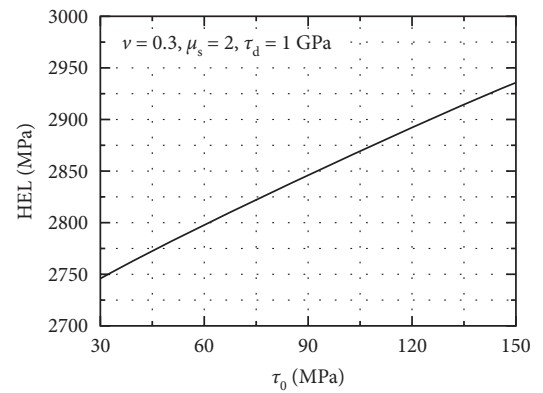

(a)

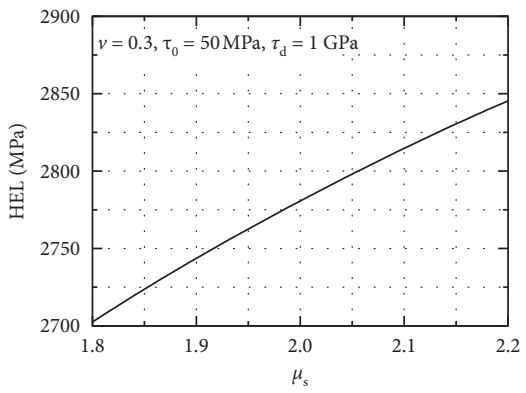

(b)

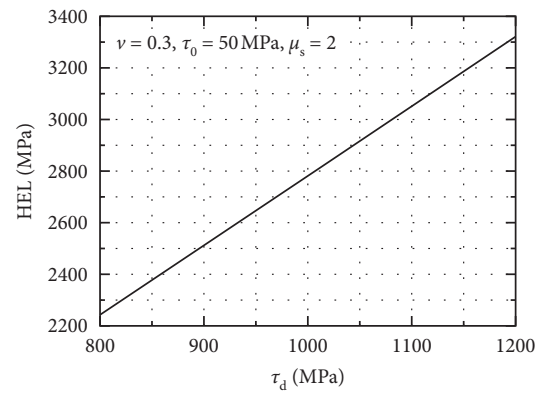

(c)

Figure 3: Sensitivity analysis of HEL with $\tau_{0}, \mu_{\mathrm{s}}$, and $\tau_{\mathrm{d}}$. (a) HEL vs. $\tau_{0}\left(\nu=0.3, \mu_{\mathrm{s}}=2, \tau_{\mathrm{d}}=1 \mathrm{GPa}\right)$. (b) HEL vs. $\mu_{\mathrm{s}}\left(\nu=0.3, \tau_{0}=50 \mathrm{MPa}\right.$, $\left.\tau_{\mathrm{d}}=1 \mathrm{GPa}\right)$. (c) HEL vs. $\tau_{\mathrm{d}}\left(\nu=0.3, \tau_{0}=50 \mathrm{MPa}, \mu_{\mathrm{s}}=2\right)$.

\section{Observation of HEL by Numerical Method}

Although the calculated HEL, as shown in Figure 2, seems to be consistent with the measured results under proper Poisson's ratio, equation (6) based on the Lundborg criterion is not verified by direct observation. In this section, finite element/difference package AUTODYN-2D is used to simulate the plate impact experiment of Li et al. [7], where a copper flyer is used to impact a granite target at an initial velocity $1.65 \mathrm{~km} / \mathrm{s}$ as shown in Figure 4(a). The mesh-free smoothed particle hydrodynamics ( $\mathrm{SPH}$ ) solver is used for the simulation, with smoothing particle size being $0.025 \mathrm{~mm}$ for all parts (Figure 4(b)).
3.1. Determination of Model Constants for Copper. The Grüneisen equation of state based on shock relation is applied for copper as shown in Table 2, while the strength of copper is ignored here.

3.2. Determination of Model Constants for Granite. The rock tested in experiments of Li et al. [7] was Wulian granite from Shandong Province in China. The microstructure of a rock sample is shown in Figure 5, and the nominal mineral composition is given in Table 3. Selected mechanical properties of the Wulian granite are shown in Table 4. 


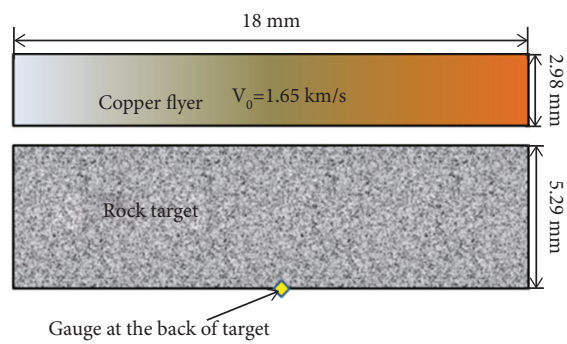

(a)

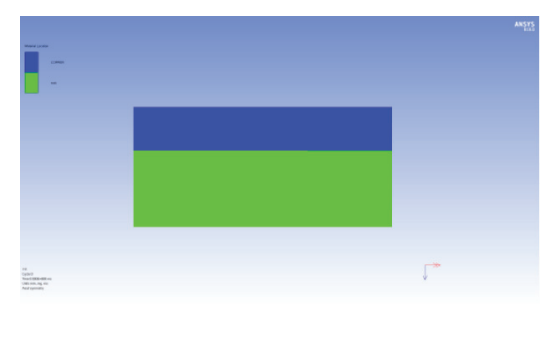

(b)

Figure 4: Design of plate impact modeling. (a) Parameters of the flyer and the target. (b) The model in AUTODYN-2D.

TABLE 2: Parameters of Grüneisen equation for copper [20].

\begin{tabular}{lccc}
\hline$\rho_{0 \mathrm{p}}$ & $\Gamma$ & $C_{1}$ & $S_{1}$ \\
\hline $8930 \mathrm{~kg} / \mathrm{m}^{3}$ & 1.99 & $3940 \mathrm{~m} / \mathrm{s}$ & $S_{1}=1.489$ \\
\hline
\end{tabular}

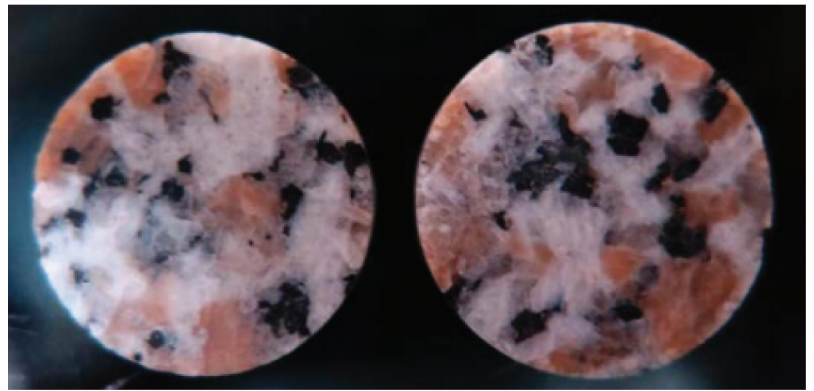

Figure 5: Granite specimen used in plate impact experiment of $\mathrm{Li}$ et al. [7].

TABLE 3: Nominal mineral composition of the Wulian granite [7].

\begin{tabular}{lc}
\hline Mineral & Wt. \% \\
\hline Plagioclase & 45 \\
Potash feldspar & 20 \\
Quartz & 20 \\
Hornblende & 10 \\
Biotite & 5 \\
\hline
\end{tabular}

TABLE 4: Selected mechanical and physical properties of the Wulian granite [7].

\begin{tabular}{lc}
\hline Property & Value \\
\hline Quasistatic tensile strength & $17 \mathrm{MPa}$ \\
Quasistatic compression strength & $89 \mathrm{MPa}$ \\
Young's modulus & $78 \mathrm{GPa}$ \\
Poisson's ratio & 0.3 \\
Density & $2670 \mathrm{~kg} / \mathrm{m}^{3}$ \\
\hline
\end{tabular}

The Lundborg criterion is inputted with a piecewise linear variation of $Y$ (i.e., $2 \tau_{\mathrm{s}}$ ) with $P$ as shown in Table 5, where $P-Y$ data are obtained with $\tau_{0}=50 \mathrm{MPa}, \mu_{\mathrm{s}}=2.0$, and $\tau_{\mathrm{d}}=1 \mathrm{GPa}$. For elastic state, $G=30 \mathrm{GPa}, K=65.1 \mathrm{GPa}$, and $\rho_{0 \mathrm{t}}=2670 \mathrm{~kg} / \mathrm{m}^{3}$ is inputted. Hence, $v$ equals 0.3 accordingly, and the HEL can be predicted to be $2.78 \mathrm{GPa}$ according to equation (6).
It should be noted that not more than 10 pairs of $P-Y$ data can be inputted in AUTODYN-2D, and if $P$ exceeds its maximum value inputted of Table 5, the strength shall obey the Tresca criterion, where $Y$ stays its maximum value. To obtain the HEL exactly with the Lundborg criterion but not the Tresca criterion, the maximum value of inputted $P$ is twice of $P_{\text {HEL }}$ predicted by

$$
P_{\mathrm{HEL}}=\frac{(\mathrm{HEL}+(2 v / 1-v) \cdot \mathrm{HEL})}{3}=1.7 \mathrm{GPa} .
$$

The comparison of the inputted data and the Lundborg criterion is shown in Figure 6.

3.3. Simulated Results. The calculation was ceased at $2.6 \mu \mathrm{s}$ after the calculation began, and the profiles of particle velocity obtained from the modeling and experiment are shown in Figure 7 . The particle velocity from modeling at the HEL $(332 \mathrm{~m} / \mathrm{s})$ is $23.7 \%$ lower than that from experiment $(435 \mathrm{~m} / \mathrm{s})$, and peak velocity from modeling $(2355 \mathrm{~m} / \mathrm{s})$ is $5.8 \%$ lower than that from experiment $(2500 \mathrm{~m} / \mathrm{s})$. From the particle velocity, the HEL can be estimated by using

$$
\mathrm{HEL}=\rho_{0 t} C_{L t} v_{\mathrm{HEL}}=\rho_{0 t} \sqrt{\frac{K+(4 / 3) G}{\rho_{0 t}}}\left(\frac{v_{r}}{2}\right)=2.78 \mathrm{GPa} .
$$

This result is consistent with the HEL by using equation (6) and close to the results by $\mathrm{Li}$ et al. $(2.36-2.63 \mathrm{GPa})$ [7]. Hence, equation (6) is proper to estimate the HEL at this point.

\section{Discussion of Some Special Effects of the HEL}

As reported by Ahrens et al. [1] and Kovalev et al. [4], amplitude decreasing of the elastic precursor is found in plate impact experiments for rock-like novaculite, quartzite, marble, and granite. The decrease in elastic shock wave amplitude with increasing propagation path length is of special significance for a plane wave system because in one dimension, the geometrically produced attenuation is absent and the decrease in elastic shock amplitude must reflect a property of the rock. On the other hand, in some cases reported by Ahrens et al. [1], a pronounced increase in the elastic shock wave amplitude with the increasing final shock state has been observed in tests of nonporous rocks and 
TABLE 5: Inputted strength data of Wulian granite based on the Lundborg criterion.

\begin{tabular}{lcccccccccc}
\hline No. & 1 & 2 & 3 & 4 & 5 & 6 & 7 & 8 & 9 & 10 \\
\hline$P(\mathrm{MPa})$ & -24 & 0 & 425 & 850 & 1275 & 1700 & 2125 & 2550 & 2975 & 3400 \\
$Y\left(=2 \tau_{\mathrm{s}}\right)(\mathrm{MPa})$ & 0 & 100 & 997 & 1318 & 1484 & 1585 & 1652 & 1701 & 1738 & 1767 \\
\hline
\end{tabular}

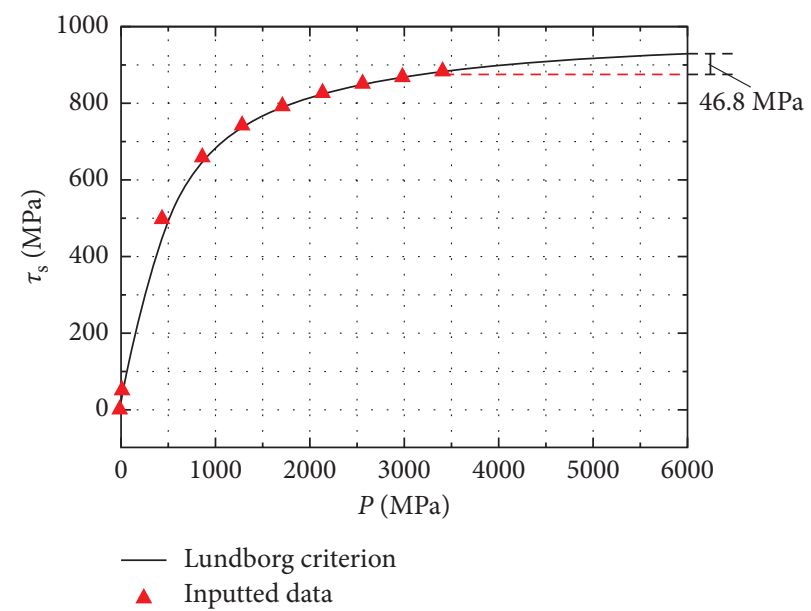

Figure 6: Comparison of the Lundborg criterion and inputted data.

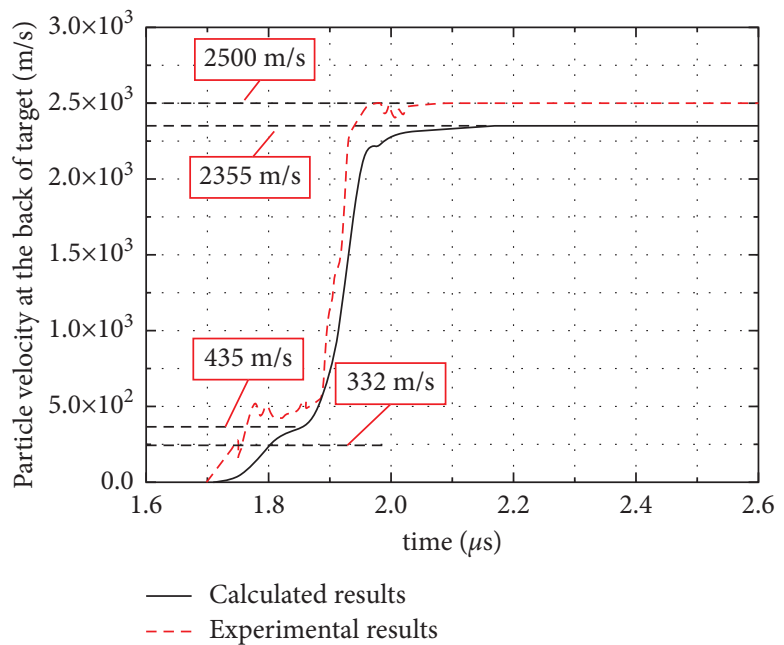

FIGURE 7: Comparison of particle velocity profiles from simulation and experiment.

minerals when the shock propagation path length was fixed. To explain these phenomena, we suppose that the "damage front" can interact with the elastic precursor and changes rock properties at the elastic precursor.

The damage front is the head of the damage zone that consists of the shocked and fractured materials [21], and it is a common consideration that because of the time delaying of the damage evolution, the damage front cannot overtake the elastic precursor (Figure 8(a)). In this study, we shall make some corrections as shown in Figure 8(b). Firstly, a diffuse and smooth front of the elastic precursor as observed by Kovalev et al. [4] and $\mathrm{Li}$ et al. [7] should be considered. Secondly, the damage front cannot overtake the leading-edge of the elastic precursor, but the damage zone and the elastic precursor partly overlap near the inflection point of the wave front. Thirdly, the HEL is an averaged result determined by the elastic precursor where damage works. Fourthly, the damage degree decays gradually from the impact point to the damage front with uneven dissipation of energy.

Based on the assumptions above, we can give a possible explanation of the connection between damage and the varying HEL. On the one hand, when the shock propagation path length was fixed, the increasing final shock state can always give rise to a higher-degree of damage, and this commonly contributes to the rising of Poisson's ratio but the reduction of shear strength. If the effect of increasing Poisson's ratio is the decisive factor, the HEL value, according to Figure 2, shall rise. On the other hand, with the 


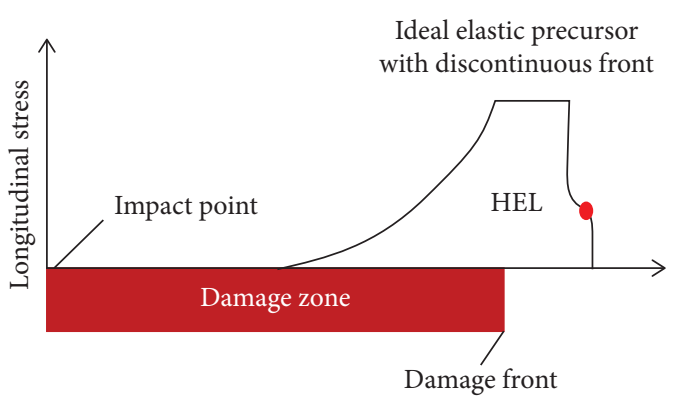

Propagation length

(a)

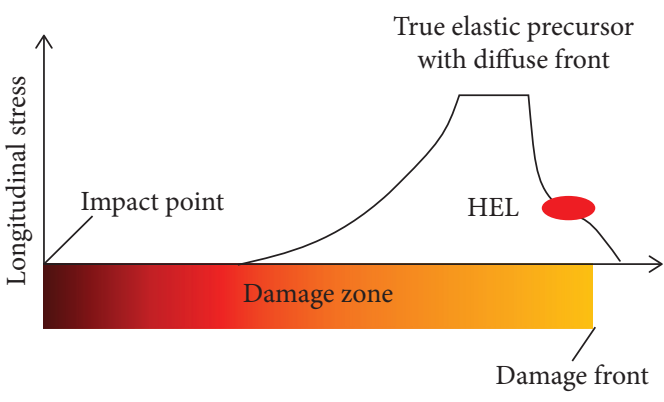

Propagation length

(b)

FIgURE 8: Two types of relative positions of elastic precursor and damage front. (a) The elastic precursor damage front is ahead of the damage front. (b) The elastic precursor with diffuse front and the damage front partly overlaps.

decaying of the damage degree along the propagation path, Poisson's ratio shall reduce to its initial value without damage accordingly. Although the recovering of the shear strength could occur simultaneously, a reduction of the HEL shall be observed if the effect of Poisson's ratio still prevails.

It should be noted that the varying Poisson's ratio with environmental parameters is rather complicated. Studies of Xing et al. [22] based on high-speed 3D-DIC show that the dynamic Poisson's ratio of Hawkesbury sandstone can undergo a wide varying from a negative value to over 0.5 . Earlier, studies of Schock et al. [19] show that effective Poisson's ratio in a uniaxial stress loading for granodiorite and the Lance sandstone can rise from 0.25 to 0.5 and 0.05 to 0.7 , respectively. The complicated evolution of Poisson's ratio is explained by Xing et al. [22] with grains rotating in the scale of rock microstructure and initiation of new microcracks or extension of existing ones. Similarly, Schock et al. [19] attributed this to the intergranular movement of microscopic clay mineral particles.

It should also be noted that some other theories about the stress relaxation behind elastic shock waves are suggested in studies of Duvall [23], where it is assumed that the shocked materials will momentarily support a value of shear stress higher than equilibrium, and as the shock front passes through an element of rock, the shear stress relaxes toward its equilibrium value, and rarefaction waves generated within the material overtake the wave front and reduce its amplitude. Nevertheless, the theories including those of this study are all helpful in studying dynamic behaviors of the rock, and more knowledge of the rock properties and damage evolution under dynamic compression should be obtained by experiments.

\section{Conclusions}

In this work, the calculation method of the HEL of hard rock is suggested based on the elastoplastic model and the Lundborg strength criterion. The results calculated are verified by those from experiments and modeling, and the influences of different parameters are compared and discussed. Mechanism of abnormal phenomena including amplitude decreasing of elastic precursor in plate impact experiments is explained. The following main conclusions can be drawn based on the results:

(1) The HEL of hard rock can be determined by the intersection of the uniaxial strain loading path with Lundborg strength criterion, and the HEL increases with Poisson's ratio, the shear strength at zero pressure, the coefficient of internal friction, and the plastic limit. Poisson's ratio seems to be the decisive factor, and the plastic limit is also important. In contrast, the effect of shear strength at zero pressure is ignorable. The environmental factors including the strain rate can influence the HEL by changing the above intrinsic properties of hard rock.

(2) The simplified model of this work can give a rational and practical prediction of the HEL of hard rock in theory. This is also verified by comparison of experimental results and modeling results by implanting the ideal elastoplastic model into AUTODYN-2D.

(3) The complicated interaction between the "damage front" and the diffuse elastic precursor is assumed to explain two special effects of the HEL in plate impact experiments of hard rock, including the decreasing of the HEL with increasing propagation path length and the increasing of the HEL with increasing final shock state. In both cases, Poisson's ratio related to damage levels seems to be the dominant factor.

\section{Notation}

$C_{1}: \quad$ Bulk sound speed

$C_{\mathrm{Lt}}: \quad$ Longitudinal sound speed

$G$ : $\quad$ Elastic shear modulus

HEL: Value of Hugoniot elastic limit

$K$ : $\quad$ Linear bulk modulus

$m: \quad$ Hoek-Brown constant

$P: \quad$ Hydrostatic pressure

$P_{\mathrm{HEL}}: \quad$ Hydrostatic pressure at Hugoniot elastic limit

$S_{1}$ : $\quad$ Slope of the shock velocity-particle velocity curve

$Y: \quad$ Yield stress

Г: $\quad$ Grüneisen coefficient 


$$
\begin{aligned}
& \mu_{\mathrm{s}}: \quad \text { Coefficient of internal friction } \\
& \nu: \quad \text { Poisson's ratio } \\
& \rho_{0 \mathrm{p}}, \rho_{0 \mathrm{t}} \text { : Initial density of impactor and target, respectively } \\
& \sigma_{x}, \sigma_{y} \text {, Longitudinal normal stress, first lateral normal } \\
& \sigma_{z}: \quad \text { stress, and second lateral normal stress, } \\
& \text { respectively } \\
& \sigma_{\mathrm{UC}}: \quad \text { Unconfined strength of the material } \\
& \tau_{\mathrm{s}}, \tau_{0} \text {, Shear strength, shear strength at zero pressure, } \\
& \tau_{\mathrm{d}}: \quad \text { and plastic limit of shear strength, respectively } \\
& v_{\mathrm{r}} \text { : } \quad \text { Rear face particle velocity. }
\end{aligned}
$$

\section{Data Availability}

Some of the data used are from papers and reports listed in references.

\section{Conflicts of Interest}

The authors declare that they have no conflicts of interest.

\section{Acknowledgments}

This research was supported by program for the National Natural Science Foundation of China (grant nos. 12002358, 12002171, 11972045, 52008391, and 51808553), China Postdoctoral Science Foundation (grant nos. 2017M621752 and 2018M643853), the Natural Science Foundation of Jiangsu Province, China (grant no. BK20190570), and Frontier Innovation Project of Army Engineering University of PLA (grant no. KYFYJQZL2005).

\section{References}

[1] T. J. Ahrens and G. E. Duvall, "Stress relaxation behind elastic shock waves in rocks," Journal of Geophysical Research, vol. 71, no. 18, pp. 4349-4360, 1966.

[2] D. E. Grady, "Shock-wave compression of brittle solids," Mechanics of Materials, vol. 29, no. 3-4, pp. 181-203, 1998.

[3] Z. Rosenberg, "On the relation between the Hugoniot elastic limit and the yield strength of brittle materials," Journal of Applied Physics, vol. 74, no. 1, pp. 752-753, 1993.

[4] A. E. Kovalev, M. N. Pavlovskii, V. M. Bel'skii, and V. V. Komissarov, "Elastoplastic behavior of marble, granite, and quartzite under shock compression," Technical Physics, vol. 71, no. 6, pp. 41-44, 2001.

[5] F. Yuan and V. Prakash, "Plate impact experiments to investigate shock-induced inelasticity in Westerly granite," International Journal of Rock Mechanics and Mining Sciences, vol. 60, pp. 277-287, 2013.

[6] J. C. F. Millett, K. Tsembelis, and N. K. Bourne, "Longitudinal and lateral stress measurements in shock-loaded gabbro and granite," Journal of Applied Physics, vol. 87, no. 8, pp. 3678-3682, 2000.

[7] G. Li, M. Y. Wang, C. M. Song, J. Li, and Y. H. Cheng, "Dynamic mechanical properties of granite by hypervelocity plate impact experiments," in Proceedings of the 6th National Conference on Engineering Safety and Protection, Xiangtan, China2018, in Chinese.

[8] E. Hoek and E. T. Brown, "Empirical strength criterion for rock masses," Journal of the Geotechnical Engineering Division, vol. 106, no. 9, pp. 1013-1035, 1980a.
[9] G. S. Collins, H. J. Melosh, and B. A. Ivanov, "Modeling damage and deformation in impact simulations," Meteoritics \& Planetary Sciences, vol. 39, no. 2, pp. 217-231, 2004.

[10] H. A. Ai and T. J. Ahrens, "Simulation of dynamic response of granite: a numerical approach of shock-induced damage beneath impact craters," International Journal of Impact Engineering, vol. 33, no. 1-12, pp. 1-10, 2006.

[11] Q. B. Zhang and J. Zhao, "A review of dynamic experimental techniques and mechanical behavior of rock materials," Rock Mechanics and Rock Engineering, vol. 47, 2013.

[12] M. Hokka, J. Black, D. Tkalich et al., "Effects of strain rate and confining pressure on the compressive behavior of Kuru granite," International Journal of Impact Engineering, vol. 91, pp. 183-193, 2016.

[13] N. Lundborg, "Strength of rock-like materials," International Journal of Rock Mechanics and Mining Science \& Geomechanics Abstracts, vol. 5, no. 5, pp. 427-454, 1968.

[14] V. V. Adushkin, A. M. Budkov, and G. G. Kocharyan, "Features of forming an explosive fracture zone in a hard rock mass," Journal of Mining Science, vol. 43, no. 3, pp. 273-283, 2007.

[15] H. B. Li, J. Zhao, and T. J. Li, "Triaxial compression tests on a granite at different strain rates and confining pressures," International Journal of Rock Mechanics and Mining Sciences, vol. 36, no. 8, pp. 1057-1063, 1999.

[16] K. Sato, M. Kawakita, and S. Kinoshita, "The dynamic fracture properties of rocks under confining pressure," Memoirs of the Faculty of Engineering, vol. 15, no. 4, pp. 467-478, 1981.

[17] Y. Yan, Y. Zheng, and H. Cao, "Strength size effect of heterogeneous rock in conventional triaxial tests," Low Temperature Architecture Technology, vol. 39, no. 12, pp. 130-133, 2017, in Chinese.

[18] C. F. Petersen, "Shock wave studies of selected rocks," vol. 99, Stanford University, California, UK, 1969, Ph.D. Thesis.

[19] R. N. Schock, H. C. Heard, and D. R. Stephens, "Stress-strain behavior of a granodiorite and two graywackes on compression to 20 kilobars," Journal of Geophysical Research, vol. 78, no. 26, pp. 5922-5941, 1973.

[20] D. Steinberg, Equation of State and Strength Properties of Selected Materials, Lawrence Livermore National Laboratory, Livermore, CA, USA, 1996.

[21] W. M. Isbell, C. E. Anderson, and J. R. Asay, Penetration Mechanics Research in the Former Soviet Union, Science Application International Corp, " San Diego, CA, USA, 1992.

[22] H. Z. Xing, Q. B. Zhang, and J. Zhao, "Stress thresholds of crack development and Poisson's ratio of rock material at high strain rate," Rock Mechanics and Rock Engineering, vol. 51, no. 3, pp. 945-951, 2018.

[23] G. E. Duvall, "Propagation of plane shock waves in a stressrelaxing medium," Stress Waves in Anelastic Solids, Springer, Berlin, Germany, pp. 20-32, 1964. 